\title{
BMJ Open Sex differences in risks of in-hospital and late outcomes after cardiac surgery: a nationwide population-based cohort study
}

\author{
Feng-Cheng Chang (D , ${ }^{1}$ Shao-Wei Chen, ${ }^{2,3}$ Yi-Hsin Chan, ${ }^{4}$ Chia-Pin Lin, ${ }^{4}$ \\ Victor Chien-Chia Wu, ${ }^{4}$ Yu-Ting Cheng, ${ }^{2}$ Dong-Yi Chen, ${ }^{4}$ Kuo-Chun Hung, ${ }^{4}$ \\ Pao-Hsien Chu (D) , ${ }^{4}$ An-Hsun Chou (D) ${ }^{1}$
}

To cite: Chang F-C, Chen S-W, Chan $\mathrm{Y}-\mathrm{H}$, et al. Sex differences in risks of in-hospital and late outcomes after cardiac surgery: a nationwide populationbased cohort study. BMJ Open 2022;12:e058538. doi:10.1136/ bmjopen-2021-058538

- Prepublication history and additional supplemental material for this paper are available online. To view these files, please visit the journal online (http://dx.doi.org/10.1136/ bmjopen-2021-058538)

F-CC and S-WC contributed equally.

Received 19 0ctober 2021 Accepted 14 January 2022

Check for updates

(c) Author(s) (or their employer(s)) 2022. Re-use permitted under CC BY-NC. No commercial re-use. See rights and permissions. Published by BMJ.

For numbered affiliations see end of article.

Correspondence to Dr An-Hsun Chou; f5455@cgmh.org.tw

\section{ABSTRACT}

Objectives Outcomes of sex differences in major cardiac surgery remain controversial. A comprehensive understanding of sex differences in major adult cardiac surgery could provide better knowledge of risk factors, management strategy and short-term or long-term outcomes. The present study aimed to investigate sex differences in the risks of outcomes of major cardiac surgeries and subgroup analyses of different valve types. Design Population-based nationwide cohort study. Setting Data were obtained from National Health Insurance Research Database (NHIRD) in Taiwan. Participants A total of 66326 adult patients (age $\geq 20$ years; $30.3 \%$ women) who underwent a first major cardiac surgery (isolated coronary artery bypass graft (CABG), isolated valve or concomitant bypass/valve) from 2000 to 2013 were identified via Taiwan NHIRD.

Main outcome measures Outcomes of primary interest were in-hospital death and all-cause mortality during follow-up period. Propensity score matching was conducted as a secondary analysis for the sensitivity test. Results Women who underwent isolated CABG tended to have greater risks of both in-hospital (OR $1.37 ; 95 \% \mathrm{Cl}$ 1.26 to 1.49 ) and late outcomes (HR $1.26 ; 95 \% \mathrm{Cl} 1.22$ to 1.31). Women after concomitant $\mathrm{CABG} /$ valve also had a greater in-hospital (OR $1.19 ; 95 \% \mathrm{Cl} 1.01$ to 1.40 ) and long-term mortality (HR $1.14 ; 95 \% \mathrm{Cl} 1.05$ to 1.24 ). Women after isolated mitral valve repair have a non-favourable outcome of in-hospital mortality (OR 1.70; $95 \% \mathrm{Cl} 1.01$ to 2.87). Women who did not receive an isolated aortic valve replacement had more favourable all-cause mortality outcome (HR $0.90 ; 95 \% \mathrm{Cl} 0.84$ to 0.96 ). Secondary analysis in the propensity score-matching cohort demonstrated results similar to the primary analysis. Conclusions Female patients who underwent procedures involving CABG (with or without concurrent valvular intervention) had generally worse outcomes. However, the results of isolated valve surgery were variable on the basis of the type of intervened valve.

\section{INTRODUCTION}

Sex differences in major cardiac surgery, including clinical presentation, disease management, complications and survival
Strengths and limitations of this study

- This study investigates the sex differences in outcomes after different major cardiac surgeries, and subgroup analyses of different valve types were also investigated.

- The follow-up course was a relatively long-term period (a maximum of 14-year time period).

- This population-based cohort study was largescale enough to analyse the outcomes we focused on by using the Taiwan National Health Insurance Research Database (NHIRD).

- Some detailed information was absent in the NHIRD, including laboratory data, echocardiographic reports, physical status and perioperative data such as types of valve prosthesis, surgical technique used and indications.

- Although this study provides adequate shortterm and long-term outcomes with properly wellcontrolled propensity score matching, the database is slightly outdated (2000 to 2013)

outcomes, have been widely studied. ${ }^{1-3}$ Nevertheless, fierce debates on sex-related differences are ongoing. Most adult cardiac procedures include coronary artery bypass graft (CABG) and single or multiple valvular surgery. ${ }^{4}$ In the past, female sex was recognised as a vulnerable factor for operation risk and prognosis. ${ }^{124}$ This theory was based on the physical frailty of female patients and profound disease severity when undergoing an operation. ${ }^{1}$

Previous published studies revealed a preference for surgical outcomes for male patients undergoing adult cardiac surgery. ${ }^{34}$ Most articles have discussed CABG, but the outcomes of valvular surgeries have been less frequently mentioned. In addition, there has been no strong agreement on the prognosis of valve surgery as compared with that of CABG..$^{5-10}$ Although some studies demonstrated the 
outcome of single-valve surgery, ${ }^{5-10}$ other conditions remain to be investigated, such as multiple-valve surgery and concomitant $\mathrm{CABG}$ and valve surgery.

A comprehensive understanding of sex differences in major adult cardiac surgery could provide better knowledge of risk factors, management strategy and short-term or long-term outcomes. The aim of the present study was to demonstrate the effects of sex differences on demographics, clinical and surgical characteristics and outcomes using information obtained from a national database in Taiwan.

\section{MATERIALS AND METHODS \\ Data source}

This retrospective, nationwide, population-based cohort study was implemented on the basis of data obtained from the Taiwan National Health Insurance Research Database (NHIRD). Covering almost $99 \%$ of the Taiwan residents, the National Health Insurance (NHI) programme was established on 1 March 1995, and NHIRD was derived from the government-operated single-payer NHI programme. The costs of all examinations (physical or imaging), laboratory tests, medications, interventional procedures and surgeries were reimbursed via Taiwan's Bureau of NHI. Because the NHIRD contains the details of major cardiac surgery, such as the type of procedure, demographic distribution and in-hospital and long-term follow-up, this database provides a practical way to analyse the outcomes in which we were interested. Constraints imposed by the Computer-Processed Personal Data Protection Law and associated NHI regulations ensured that the investigators' access to the database was limited to research purposes only. Detailed information about the NHIRD is included in previous articles. ${ }^{11}$

\section{Study population}

Using the Taiwan NHI reimbursement codes, we identified hospitalisation records with any cardiac surgeries (isolated $\mathrm{CABG}$, isolated valve or CABG +valve) between 1 January 2000 and 31 December 2013. For patients with multiple cardiac surgeries, the first cardiac surgery during the study period was assigned as the index surgery. Patients with missing demographics and those aged $<20$ years were excluded. After selection, 66326 patients were eligible for analysis. These patients were subsequently categorised into the following three groups: isolated CABG, isolated valve surgery and concomitant CABG and valve surgery. All three groups were further stratified into male and female patients (figure 1).

\section{Comorbidities}

Covariates included age, monthly income, urbanisation level, history of previous cardiac surgery, presence of urgent surgery, 10 comorbidities (diabetes mellitus, hypertension, heart failure, coronary arterial disease, prior myocardial infarction, peripheral arterial disease, prior stroke, chronic obstructive pulmonary disease, liver cirrhosis and malignancy), Charlson Comorbidity Index score, hospital level of the index cardiac surgery, cumulative hospital volume of cardiac surgeries (CABG or valve) between 2000 and 2013, surgical type (isolated $\mathrm{CABG}$, isolated valve or $\mathrm{CABG}+$ valve), surgical details of $\mathrm{CABG}$ and valve surgeries, type of valve surgery and

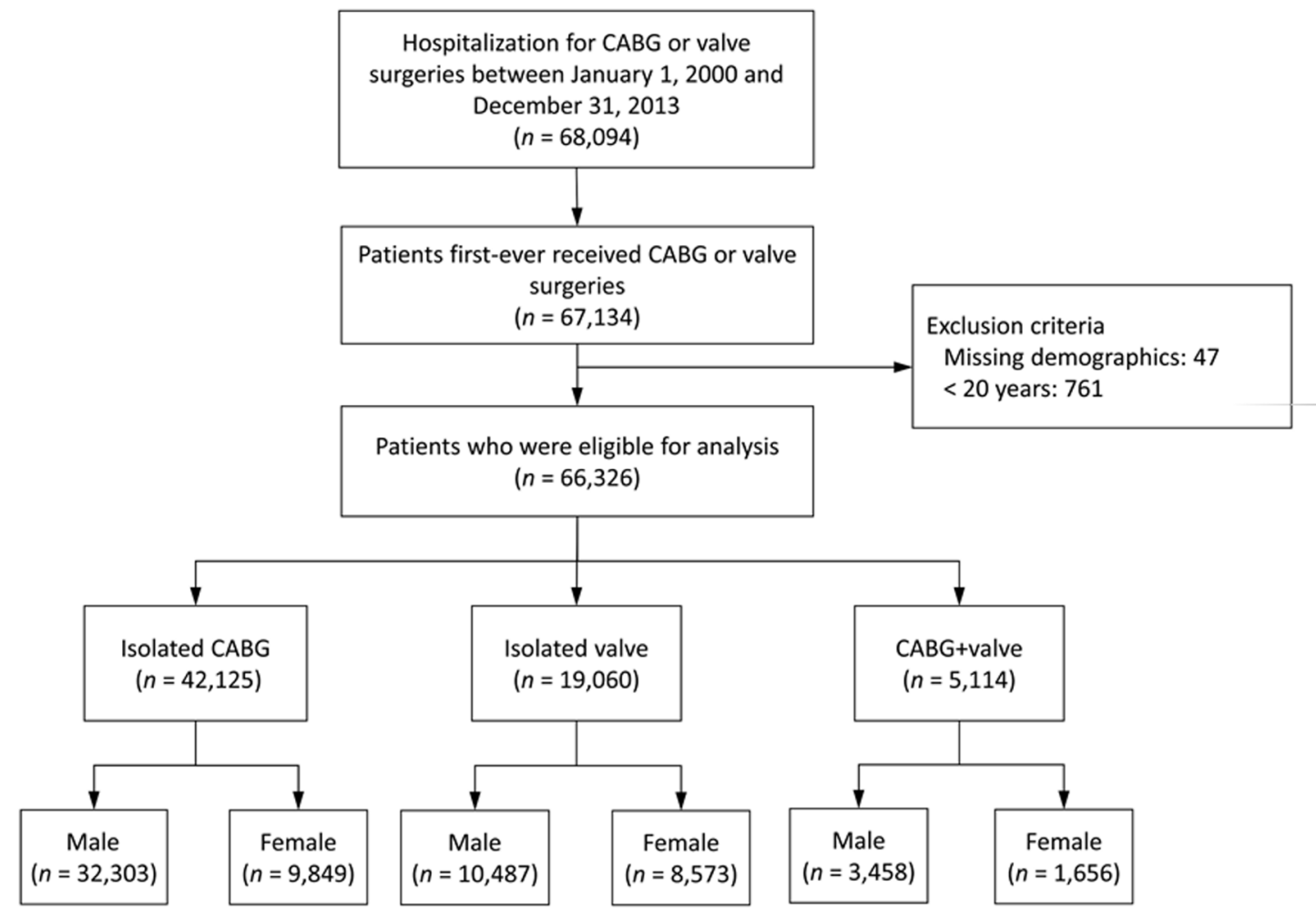

Figure 1 Flowchart of patient selection. CABG, coronary artery bypass graft. 
concomitant maze procedure. The International Classification of Diseases, 9th Revision, Clinical Modification (ICD-9-CM), diagnostic codes were used to detect diseases (online supplemental table 1). Comorbidities were identified using at least two outpatient diagnoses or any single inpatient diagnosis in the previous year of the index cardiac surgery. Previous cardiac surgery, surgical details and concomitant surgeries were detected using the Taiwan NHI reimbursement codes from the inpatient claims data. The type of valve surgery was identified using ICD-9-CM procedural codes (online supplemental table 2) ${ }^{12-14}$

\section{Outcomes}

Outcomes included both in-hospital (perioperative) complications and late outcomes. In-hospital outcomes were in-hospital mortality, new-onset stroke and newonset dialysis during the admission for the index surgery. Late outcomes were all-cause mortality, major adverse cardiovascular events (MACE) and all-cause readmission during follow-up. Death was defined as a withdrawal from the NHI program. ${ }^{15}$ MACE included any one of the following: cardiovascular death, revascularisation, acute myocardial infraction or ischaemic stroke during follow-up. Cardiovascular death was considered when the criteria of the Standardized Definitions for Cardiovascular and Stroke Endpoint Events in Clinical Trials by the US Food and Drug Administration in the USA were met. The occurrence of acute myocardial infraction and ischaemic stroke was defined as the principal diagnosis of admission. Revascularisation was identified using the Taiwan NHI reimbursement codes of inpatient claims data. The ICD-9-CM and NHI codes were provided in the supplement (online supplemental table 2). For the analysis of late outcome, each patient was followed from the index cardiac surgery to the date of death, the date of outcome occurrence or 31 December 2013, whichever came earlier.

\section{STATISTICAL ANALYSIS}

We tested the trend of sex distribution in receiving isolated $\mathrm{CABG}$ or isolated valve surgeries across the study period, using the Cochran-Armitage $\chi^{2}$ test. The primary analysis was performed to compare sex differences in the risks of outcomes using the raw data for real-world practice without propensity score matching (PSM). The secondary analysis was based on the propensity scorematched cohort. The propensity score was the predicted probability to be woman given the values of covariates (except for follow-up year, in-hospital and late outcomes) using the multivariable logistic regression without considering interaction effects. The follow-up year is related to long-term outcomes and, therefore, the admission date of the index surgery was included in the propensity score calculation instead. Each woman was matched to a man. PSM was conducted separately for different surgical types: isolated $\mathrm{CABG}$, isolated valve and CABG combined with valve. The matching was processed using a greedy nearest neighbour algorithm with a calliper of 0.2 times the SD of the logit of the propensity score, with random matching order and without replacement. Sex differences in demographics as well as clinical and surgical characteristics were assessed using the standardised difference, in which an absolute value of the standardised difference $<0.1$ was considered a negligible difference, and a value ranging from 0.1 to 0.2 was considered a small difference.

Sex differences in in-hospital outcomes were compared using logistic regression analysis for the binary outcome. Using the Cox proportional hazard model, we compared sex differences in the risks of fatal late outcomes (ie, all-cause mortality and MACE). The sex differences in the risks of time to nonfatal late outcomes (ie, all-cause readmission) were compared using the Fine and Gray subdistribution hazard model, which considered all-cause mortality a competing risk. In the secondary analysis based on the PSM cohort, the within-pair clustering of outcomes among the matched pairs was accounted for using a robust $\mathrm{SE}$ in all of the aforementioned regression analyses.

To focus on the population of isolated valve surgery, we performed a subgroup analysis according to several prespecified subgroup variables: isolated aortic valve replacement (AVR), isolated mitral valve (MV) repair, isolated MV replacement and number of intervened valves on in-hospital mortality and all-cause mortality. The subgroup analysis was conducted for both the raw data for real-world practice and the PSM cohort. The aforementioned subgroup analysis was performed based on interaction models. A two-sided $\mathrm{p}$ value $<0.05$ was considered statistically significant. However, the Bonferroni adjustment for type I error inflation was also made due to there were six outcomes in this study, and, therefore, the corrected significance was $p$ value $<0.0083$. Statistical analyses were performed using SAS V.9.4 (SAS Institute, Cary, North Carolina).

\section{Patient and public involvement}

No patient was involved in this study.

\section{RESULTS \\ Epidemiology of isolated cardiac surgery in Taiwan}

Among the population with isolated cardiac surgery, the proportion of female patients decreased gradually over the study period ( $\mathrm{p}$ for trend=0.004; figure $2 \mathrm{~A}$ ). On the other hand, among the population receiving isolated valve surgery, the proportion of female patients increased gradually across the years ( $\mathrm{p}$ for trend $=0.073$; figure $2 \mathrm{~B}$ ).

\section{Baseline characteristics}

Table 1 and online supplemental table 3 summarise the baseline characteristics between sexes after combining the data of different cardiac surgeries. Women tended to be slightly older, had lower socioeconomical status and had more comorbid conditions such as diabetes mellitus and 
A

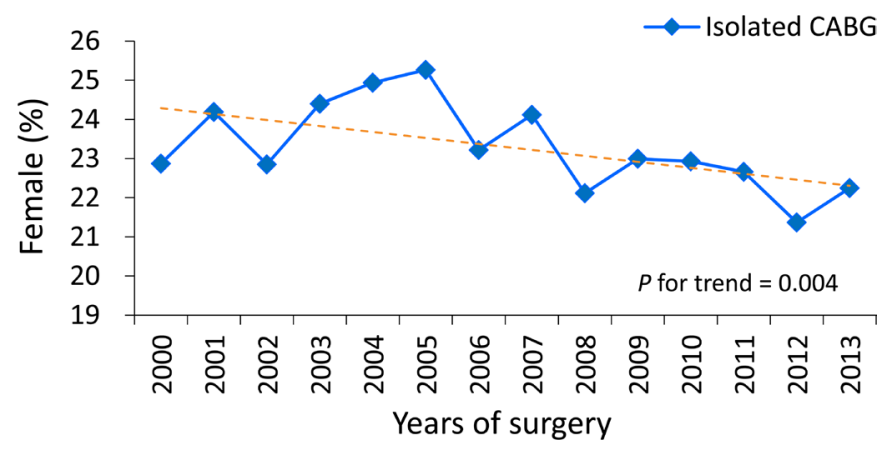

B

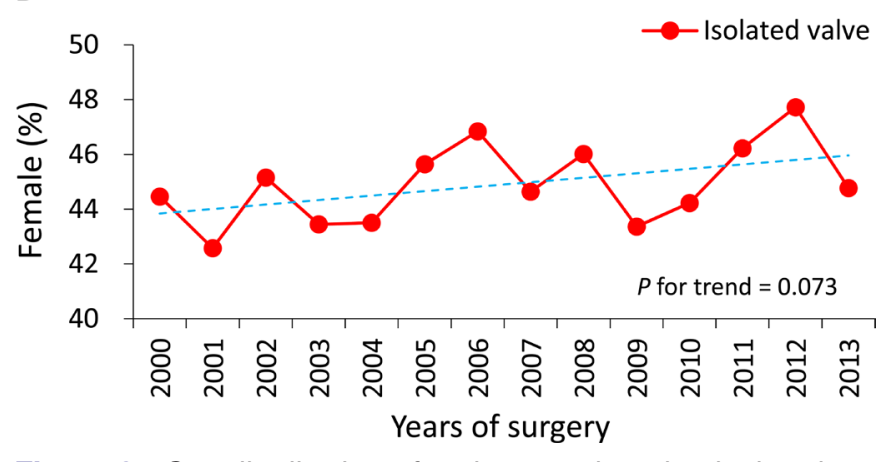

Figure 2 Sex distribution of patients undergoing isolated CABG $(A)$ and isolated valve surgeries $(B)$ across the study years in Taiwan. CABG, coronary artery bypass graft.

heart failure. Male patients had more vascular-associated conditions such as coronary arterial disease and prior myocardial infarction. Male patients were more likely to undergo isolated CABG surgery, whereas female patients had more isolated valve surgeries. Among patients who underwent $\mathrm{CABG}$, women had a lower number of bypassed vessels $(2.8 \pm 0.8)$. With regards to valve surgeries, female patients tended to have a greater number of intervened valves (1.5 \pm 0.6$)$. Men more frequently underwent aortic valve repair or replacement as compared with women. Female patients more frequently received MV replacement and tricuspid repair than male patients did. Finally, all sex differences in patient baseline characteristics were negligible after PSM. The detailed complete version of table 1 is provided as online supplemental table 3 . Online supplemental tables 4-6 list the detailed baseline characteristics between sexes for each surgical type both before and after PSM.

\section{In-hospital (short-term) outcomes}

Table 2 demonstrates the sex difference of the in-hospital outcomes before and after PSM. Among patients who received isolated CABG, women tended to have a greater risk in in-hospital mortality $(8.8 \%$ vs $6.5 \%$; OR $1.37,95 \%$ CI 1.26 to $1.49 ; \mathrm{p}<0.001)$. Besides, women after $\mathrm{CABG}$ also had higher rate of new-onset stroke and dialysis during admission. In contrast, among the population who underwent isolated valve surgery, women had a lower risk of new-onset stroke during admission. Nevertheless, women had slightly higher risks of in-hospital mortality (6.8\% vs $6.2 \%$; OR 1.10 , 95\% CI 0.98 to 1.24 ; $\mathrm{p}=0.094$ ) among those undergoing isolated valve surgery, although not statistically significant. Among patients who had combined CABG and valve surgery, women had greater risks of in-hospital mortality $(15.8 \%$ vs $13.6 \%$; OR $1.19,95 \%$ CI 1.01 to $1.40 ; \mathrm{p}=0.036$ ) and new-onset dialysis during admission. Secondary analysis in the PSM cohort revealed less significant results as compared with the primary analysis because of the smaller sample size. Noticeably, several results turned to be insignificant after adjustment for type I error inflation.

\section{Late follow-up (long-term) outcomes}

Table 3 presents the comparison of sex differences in the long-term outcomes for each surgical type using realworld data before and after PSM. Among patients who received isolated CABG, women tended to have a greater risk in all-cause mortality $(44.8 \%$ vs $37.5 \%$; HR 1.26 , $95 \%$ CI 1.22 to $1.31 ; \mathrm{p}<0.001)$, all-cause readmission and MACE during late follow-up. Nevertheless, women who underwent isolated valve surgery had a lower risk of allcause mortality (29.2\% vs $31.6 \%$; HR $0.92,95 \%$ CI 0.87 to $0.97 ; \mathrm{p}=0.001$ ) and MACE during follow-up. Finally, among patients who had concurrent CABG and valve surgery, women had greater risks of all-cause mortality during follow-up (52.5\% vs $47.6 \%$; HR 1.14, $95 \%$ CI 1.05 to $1.24 ; \mathrm{p}=0.002)$. Secondary analysis in the PSM cohort demonstrated results similar to the primary analysis. However, it is noted that several results turned to be insignificant after adjustment for type I error inflation.

\section{Subgroup analysis of mortality outcomes}

To focus on the population who received isolated valve surgery, we performed subgroup analysis on in-hospital (short-term) mortality and all-cause mortality (longterm). Sex differences in in-hospital mortality were more apparent when isolated MV repair ( $p$ for interaction=0.088) was conducted. Female patients tended to have non-favourable in-hospital mortality (HR 1.70; 95\% CI 1.01 to 2.87) when undergoing isolated MV repair (figure 3A). The secondary analysis under the PSM cohort revealed similar but less significant results as compared with the primary analysis (figure $3 \mathrm{~B}$ ).

On the other hand, sex differences in all-cause mortality were more obvious when isolated AVR ( $p$ for interaction $=0.091$ ) was conducted. Female patients tended to have a more favourable outcome of all-cause mortality (HR 0.90; 95\% CI 0.84 to 0.96) when isolated AVR was not undergone (figure 4A). The secondary analysis in the PSM cohort demonstrated similar results as that of the primary analysis (figure 4B).

\section{DISCUSSION}

\section{Main findings}

In both the group undergoing isolated $\mathrm{CABG}$ and concomitant $\mathrm{CABG}$ /valve surgery, female patients had a higher rate of in-hospital mortality during admission and 
Table 1 Brief demographics, clinical and surgical characteristics of the female and male patients

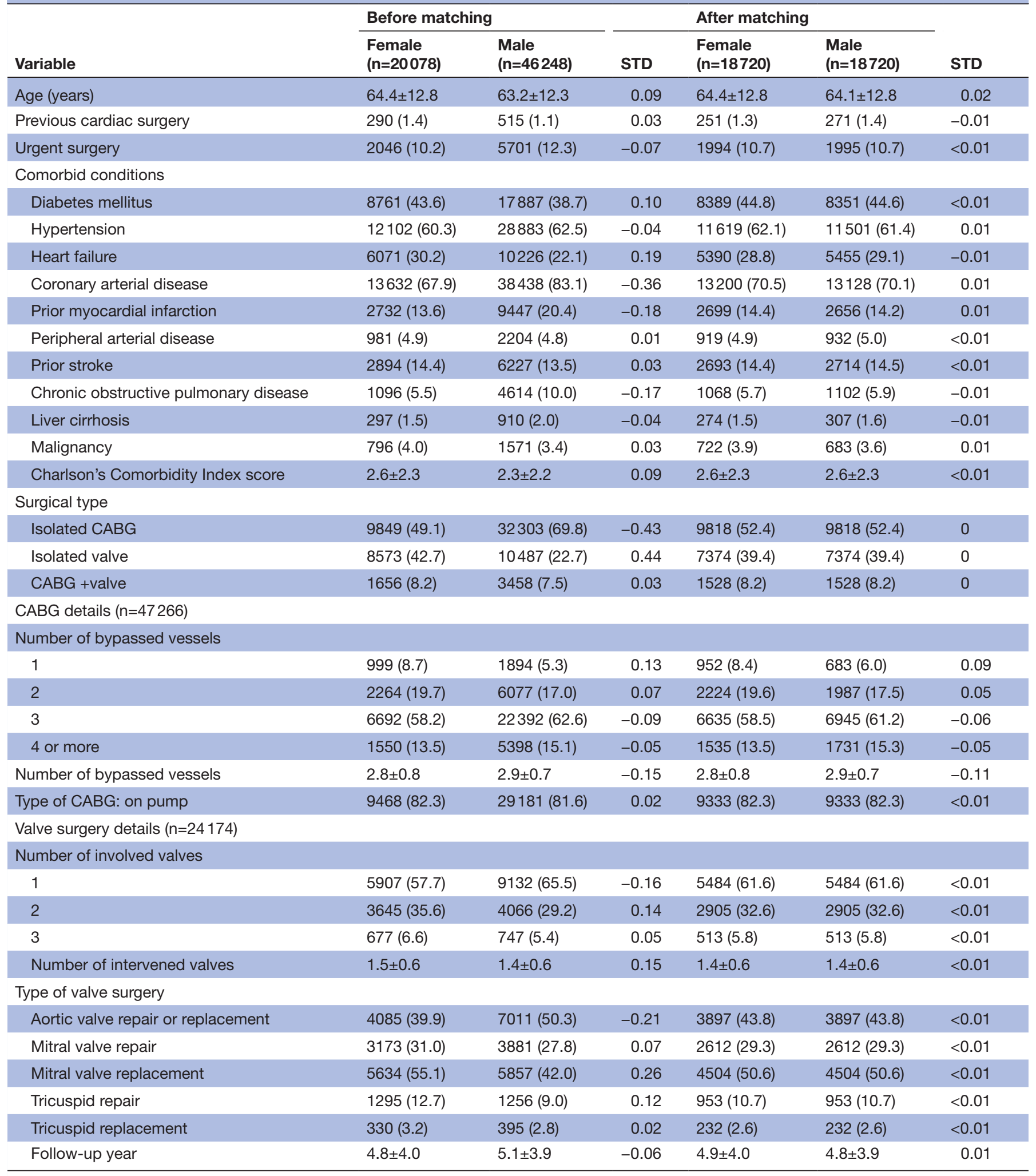

Values are given as number (\%) or mean \pm SD.

CABG, coronary artery bypass graft; STD, standardised difference.

all-cause mortality during follow-up. In terms of complications, female patients undergoing CABG tended to suffer from new-onset stroke, dialysis and even MACE. Second, female patients undergoing isolated valve surgery had better outcomes in all-cause mortality during follow-up and fewer complications such as new-onset stroke and MACE, although their rate of in-hospital mortality was slightly higher than that of men. After obtaining real-world 
Table 2 In-hospital outcomes of the female versus male patients before and after propensity score matching

\begin{tabular}{|c|c|c|c|c|}
\hline Cohort/outcome/surgical type & Female & Male & OR/HR/SHR for female $(95 \% \mathrm{Cl})$ & $\mathbf{P}$ \\
\hline \multicolumn{5}{|l|}{ Before matching } \\
\hline \multicolumn{5}{|c|}{ In-hospital mortality during admission } \\
\hline Isolated CABG & $862(8.8)$ & $2111(6.5)$ & 1.37 (1.26 to 1.49$)$ & $<0.001^{*}$ \\
\hline Isolated valve & $582(6.8)$ & $649(6.2)$ & 1.10 (0.98 to 1.24$)$ & 0.094 \\
\hline $\mathrm{CABG}+$ valve & $262(15.8)$ & $471(13.6)$ & 1.19 (1.01 to 1.40$)$ & 0.036 \\
\hline \multicolumn{5}{|c|}{ New onset stroke during admission } \\
\hline Isolated CABG & $332(3.4)$ & $872(2.7)$ & 1.26 (1.11 to 1.43$)$ & $0.001^{*}$ \\
\hline Isolated Valve & $241(2.8)$ & $352(3.4)$ & $0.83(0.71$ to 0.98$)$ & 0.031 \\
\hline CABG+valve & $65(3.9)$ & $136(3.9)$ & $1.00(0.74$ to 1.35$)$ & 0.989 \\
\hline \multicolumn{5}{|c|}{ New onset dialysis during admission } \\
\hline Isolated CABG & $974(9.9)$ & $2383(7.4)$ & 1.38 (1.28 to 1.49$)$ & $<0.001^{*}$ \\
\hline Isolated valve & $506(5.9)$ & $599(5.7)$ & 1.04 (0.92 to 1.17$)$ & 0.575 \\
\hline $\mathrm{CABG}+$ valve & $264(15.9)$ & $414(12.0)$ & 1.40 (1.18 to 1.65$)$ & $<0.001^{*}$ \\
\hline \multicolumn{5}{|l|}{ After matching } \\
\hline \multicolumn{5}{|c|}{ In-hospital mortality during admission } \\
\hline Isolated CABG & $853(8.7)$ & $770(7.8)$ & $1.12(1.01$ to 1.24$)$ & 0.031 \\
\hline Isolated Valve & $500(6.8)$ & $422(5.7)$ & 1.20 (1.05 to 1.37$)$ & $0.008^{*}$ \\
\hline CABG+valve & $234(15.3)$ & $227(14.9)$ & $1.04(0.85$ to 1.26$)$ & 0.723 \\
\hline \multicolumn{5}{|c|}{ New onset stroke during admission } \\
\hline Isolated CABG & $330(3.4)$ & $297(3.0)$ & $1.11(0.95$ to 1.31$)$ & 0.182 \\
\hline Isolated valve & $203(2.8)$ & $254(3.4)$ & 0.79 (0.66 to 0.96$)$ & 0.016 \\
\hline CABG+valve & $57(3.7)$ & 67 (4.4) & 0.84 (0.58 to 1.22$)$ & 0.370 \\
\hline \multicolumn{5}{|c|}{ New onset dialysis during admission } \\
\hline Isolated CABG & $966(9.84)$ & $967(9.85)$ & $1.00(0.91$ to 1.10$)$ & 0.981 \\
\hline Isolated valve & $419(5.7)$ & $421(5.7)$ & $1.00(0.87$ to 1.14$)$ & 0.943 \\
\hline CABG+valve & 228 (14.9) & 187 (12.2) & 1.26 (1.02 to 1.55$)$ & 0.031 \\
\hline
\end{tabular}

*Significant after Bonferroni adjustment for type I error inflation $(p<0.0083)$.

CABG, coronary artery bypass graft; SHR, subdisribution HR.

data, we demonstrated a PSM to determine whether sex was a predictor of surgical outcomes. Matching revealed that the findings were generally similar to the primary analysis in the three groups. Finally, we performed the subgroup analysis to investigate the details of the outcomes of different intervened single valvular procedures. Female patients tended to have non-favourable in-hospital mortality after undergoing isolated MV repair. The outcomes of all-cause mortality in women who did not undergo isolated AVR were better as compared with those in men.

\section{Sex differences in procedures including coronary bypass surgery}

This present study reveals that both isolated CABG and concomitant $\mathrm{CABG} /$ valve surgery were associated with worse early and late outcomes in women. This result could be mainly attributed to the involvement of coronary bypass surgery. Female patients undergoing coronary surgical bypass are older, have a smaller body surface area, have more underlying comorbidities and have a greater need for urgent or emergent surgical intervention. ${ }^{316}{ }^{17}$ Female patients more frequently present with atypical or non-anginal symptoms, which likely contributes to delays in the diagnosis, treatment and recognition of surgical coronary disease. ${ }^{1718}$ Moreover, the worse clinical outcomes can be explained by factors such as smaller coronary arteries, fewer internal mammary artery grafts, fewer bypass grafts and graft-coronary mismatch. ${ }^{17}{ }^{19} \mathrm{In}$ addition, the fear of surgery may result in delayed referral for CABG in female patients. ${ }^{1}$ Figure 2 shows that the number of female patients undergoing isolated CABG are decreasing. This fact might be related to the advancement in percutaneous coronary intervention procedures, resulting in a decrease in referral to CABG for female patients who are older, are frail, or have multiple comorbidities. ${ }^{4}$ A recent analysis reported by Soham et al demonstrated that women after CABG surgery had greater risk of in-hospital death and 30-day and 90-day readmission compared with men. ${ }^{17}$ They also described that women continue to have worse outcomes even though the 
Table 3 Long-term outcomes of the female versus male patients before and after propensity score matching

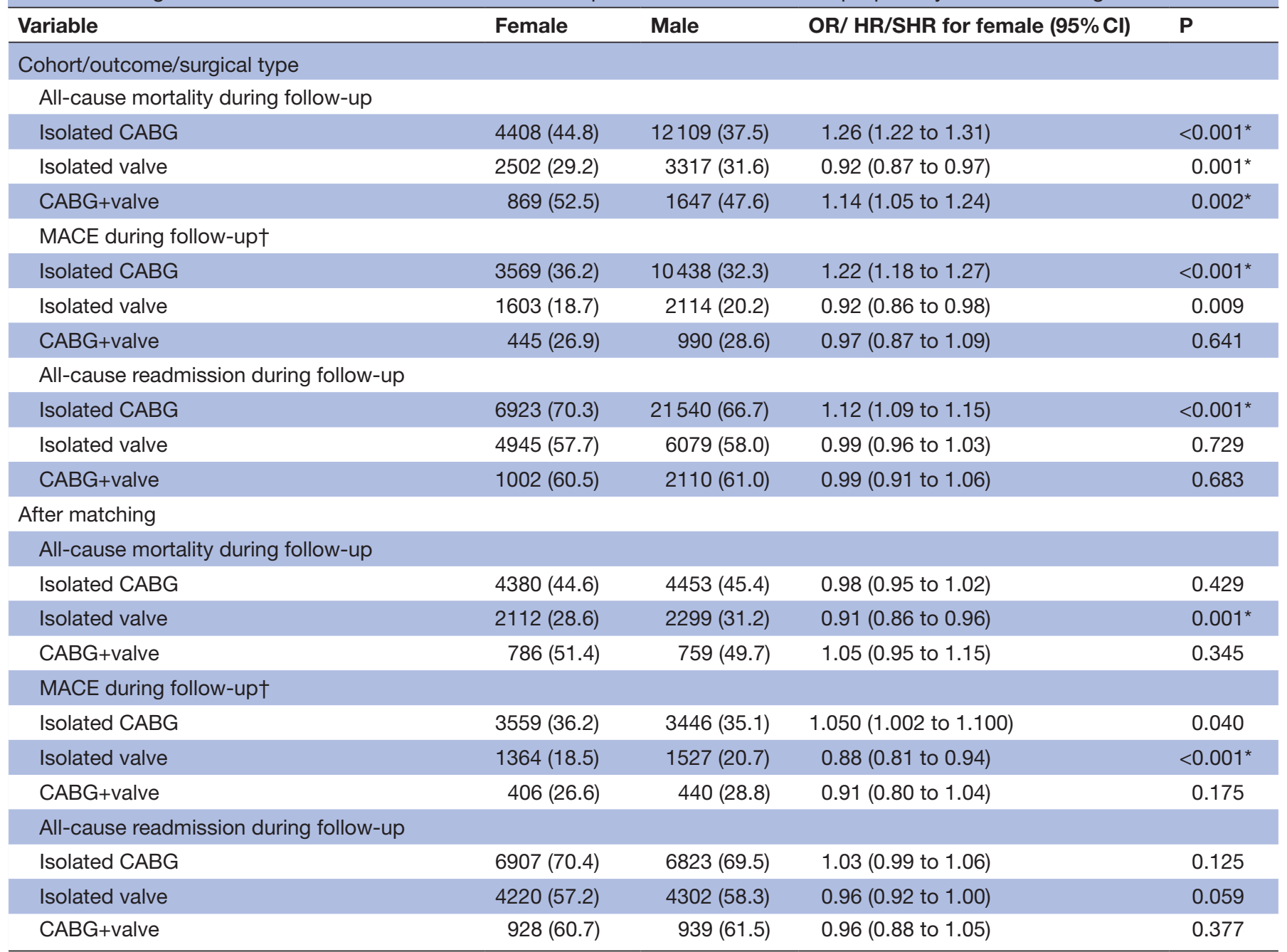

*Significant after Bonferroni adjustment for type I error inflation $(\mathrm{p}<0.0083)$.

†Including anyone of cardiovascular death, revascularisation, acute myocardial infraction or ischaemic stroke.

CABG, coronary artery bypass graft; MACE, major adverse cardiovascular events; SHR, subdisribution HR.

current clinical practice has been modified to reduce sex difference. And these points and finding supported our study results.

Few studies have investigated the outcomes of sex differences after combined CABG/valve surgery. ${ }^{20-22}$ As compared with the better knowledge of outcomes of isolated CABG, there is no strong agreement on whether there are sex differences in the outcomes of combined CABG/valve surgery. In this study, the data between isolated $\mathrm{CABG}$ and combined $\mathrm{CABG} /$ valve surgery were similar. These data indicate the increase in morbidity and mortality and relate to late presentation or referral. Therefore, the worse outcomes among female patients undergoing simultaneous CABG/valve surgery could be explained by the involvement of coronary bypass surgery rather than the valve operation itself. Earlier studies comparing the outcomes of different types of valve surgery (single or multiple) with or without concurrent CABG also support this perspective. ${ }^{20-23}$ Sinead et al recently reported their analysis that women undergoing major cardiac surgery had much more chance to receive allogeneic transfusions compared with men, especially in non-White races. Moreover, allogeneic transfusions were associated with a higher likelihood of in-hospital mortality. ${ }^{24}$

\section{Sex differences in intervened valve procedure}

As mentioned in the previous section, female sex may not always be a vulnerable factor of surgical outcomes. Our study results indicated that women undergoing isolated valve surgeries had lower rates of all-cause mortality, newonset stroke during admission and MACE. However, there are few published articles based on a retrospective large population data to clarify this issue. Johnston $e t a \hat{l}$ found no significant difference in 30-day mortality or in longterm follow-up after isolated valve surgery (single-valve and multiple-valve surgery). The isolated valve surgeries received by patients were composed of different types of intervened valves, and some involved more than one 

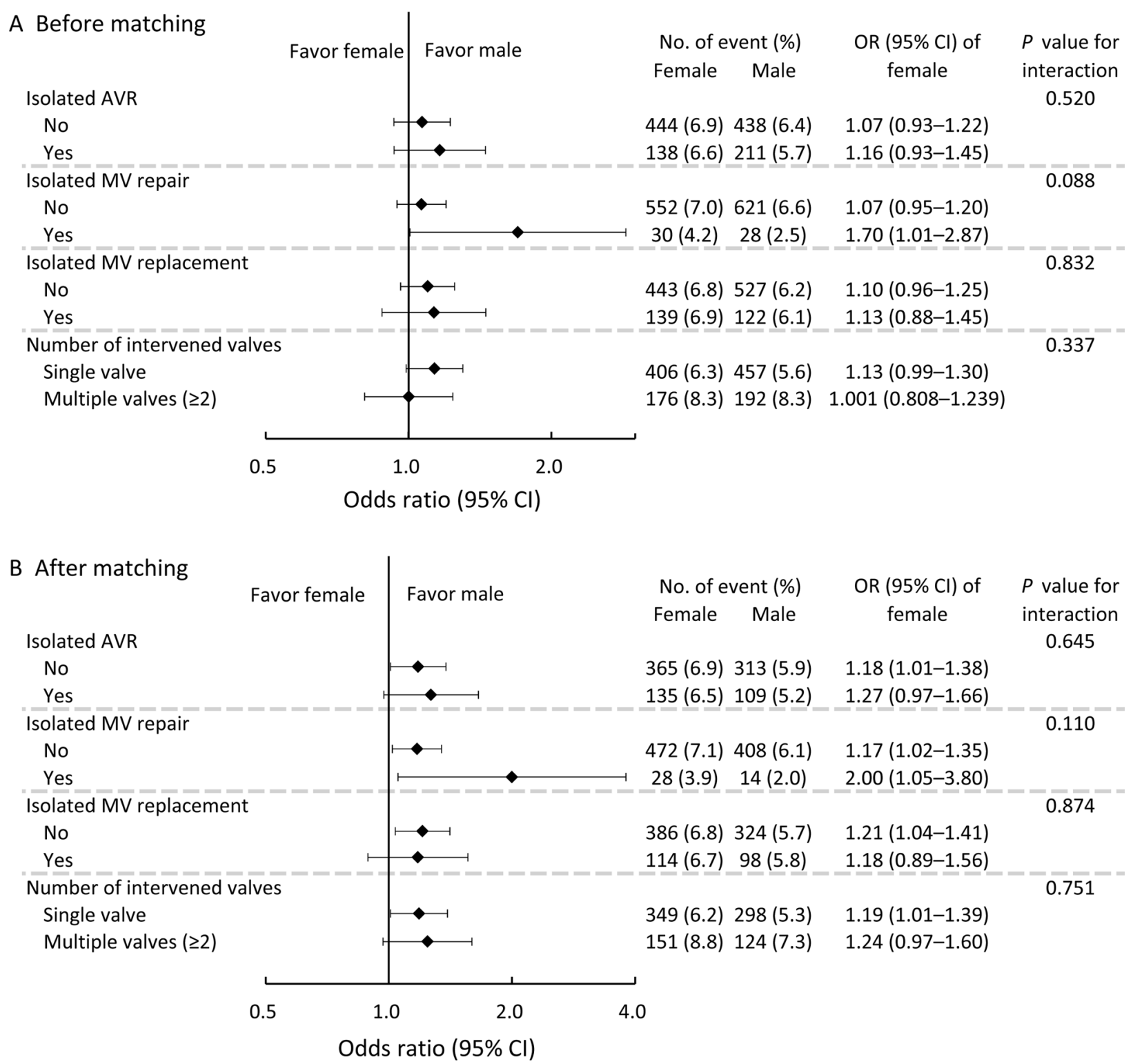

Figure 3 Subgroup analysis of the comparison of sex differences in the risks of in-hospital mortality using the whole data (A) or the propensity score-matched cohort (B). AVR, aortic valve replacement; MV, mitral valve.

valve. Therefore, the different types of valve surgery were stratified for subgroup analysis in this study.

There was no obvious sex difference in in-hospital mortality among patients undergoing isolated AVR. In addition, female patients tended to have more favourable all-cause mortality when they did not receive isolated AVR. The clinical outcomes of isolated AVR between women and men are controversial, without strong consensus. An earlier study by Aranki $e t a l^{20}$ found that sex was not a predictor of operative mortality among those undergoing isolated AVR. Mokhles et $a l^{8}$ also reported that men and women had comparable in-hospital mortality (OR 1.20, $95 \%$ CI 0.90 to $1.61 ; p=0.220$ ). Nevertheless, some studies have demonstrated that women have worse in-hospital mortality following AVR as compared with men ${ }^{5}$ or even higher late mortality. ${ }^{6}$ These findings, therefore, provide a perspective of advocating for transcatheter AVR in women with a lower threshold. A recent retrospective article showed no difference in overall survival between women and men after adjusting for preoperative risk factors ${ }^{25}$ which supports our results after PSM. Female sex itself may not be a predictor in isolated AVR, and it could be related to the worse perioperative profile of women.

In this present study, female patients underwent MV replacement more frequently than male patients did. The number of patients receiving MV repair were comparable between women and men. Our data show that female patients undergoing isolated MV repair had higher rates of in-hospital mortality but not all-cause mortality. The outcomes of MV replacement in early and late mortality were comparable between women and men. On the other hand, Johnston et al documented that male patients undergoing MV repair had higher rates of long-term survival, whereas female patients undergoing MV replacement had higher rates of long-term survival. 


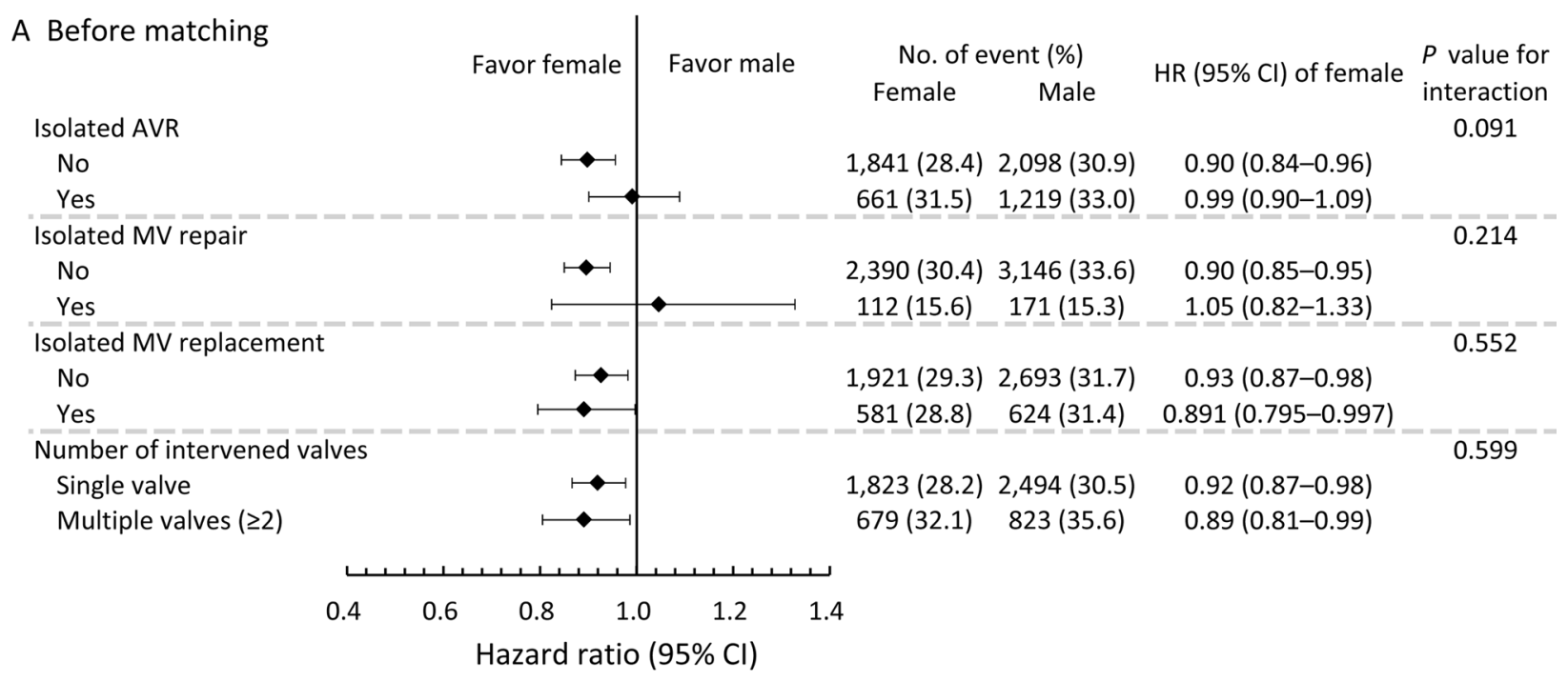

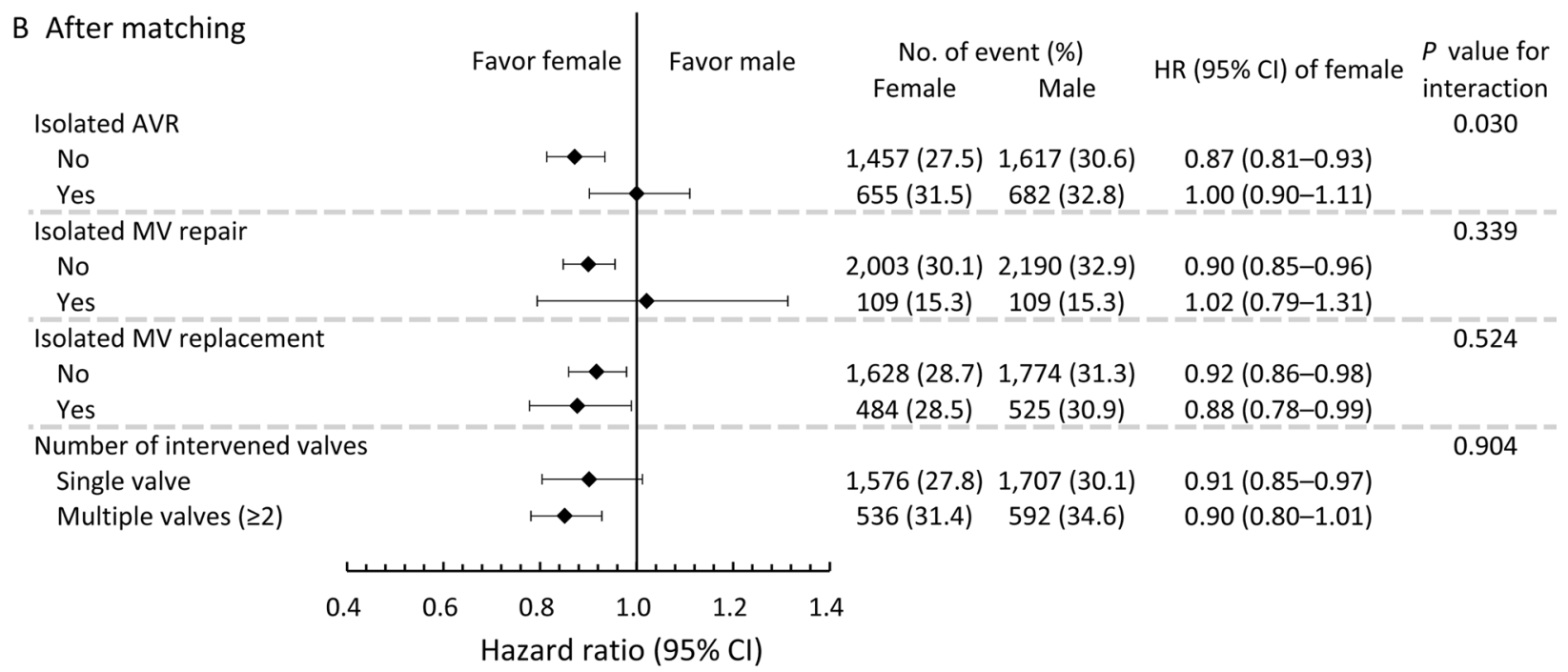

Figure 4 Subgroup analysis of the comparison of sex differences in the risks of all-cause mortality during follow-up using the whole data (A) or the propensity score-matched cohort (B). AVR, aortic valve replacement; MV, mitral valve.

Another recent nationwide cohort study reported no significant differences between men and women in-hospital mortality with both MV repair and MV replacement. $^{7}$ In contrast, Vassileva et $a l^{26}$ found a higher mortality rate in female patients undergoing MV surgery. Because there is no strong agreement of the outcomes on isolated MV surgery, further research is needed. As with other cardiac surgical procedures, female patients who underwent isolated MV surgery were older and had a more profound disease severity when presenting for the operation. ${ }^{7}$ Studies have reported a delayed detection of severe MV disease and late referral to surgical intervention in women. ${ }^{27} 28$ This is compatible with the current condition of female patients in Taiwan, who have relatively low socioeconomic status and are afraid to undergo surgery. A delay in surgery might partially explain our results of higher in-hospital mortality in female patients, and female sex may not to be a predictor of long-term outcomes of isolated MV surgery.

\section{Limitations}

Because this current study is inherent to the NHIRD, some detailed information was absent, including physical examination, vital signs, laboratory data, echocardiographic reports and heart failure symptoms. Some intraoperative and postoperative data were also not able to be retrieved from the database such as length of cardiopulmonary bypass, types of valve prosthesis, surgical technique used, indications, perioperative use of inotropes or intra-aortic balloon pump and postoperative cardiogenic shock. ${ }^{17}$ Besides, although we performed a largescale nationwide cohort, the results may not represent generalisation to other Asian or non-Asian populations. ${ }^{17}$ In addition, misclassification and coding errors might have occurred during the analysis of the NHIRD data, which is based on ICD-9-CM codes. Nevertheless, the NHI programme has strict regulations regarding surgery, examination and medication reimbursements. Comprehensive reviews of all medical records including imaging 
studies and laboratory data ensure that specific examinations or interventions are granted only to patients who are indicated. Therefore, the use of Taiwan's Taiwan NHI reimbursement codes for major cardiac-related diagnoses and procedures is precise and has been validated. ${ }^{29}$ Despite this study provides short-term and longterm outcomes with properly well-controlled PSM, the database is slightly outdated (2000 to 2013), and clinical practice may have changed in past years. Nevertheless, our study focused on the long-term outcomes of sex difference, and the sex-based disparity is inherent. Gupta et $a l^{17}$ documented their recent study results and revealed that despite efforts in reducing the sex disparity, women still have worse outcomes in current era. Therefore, we assume that the extension of the follow-up period may have limited impact to our conclusion. Finally, although medical costs might be important in some regions where health insurance is not readily available, we did not calculate these costs.

\section{Conclusions}

We conducted a nationwide, population-based cohort study to determine the sex-related differences in clinical outcomes after major adult cardiac surgery. Female patients who underwent procedures involving coronary bypass surgery (with or without concurrent valvular intervention) generally had worse outcomes. However, the results of isolated valve surgery were variable on the basis of the type of the intervened valve. Understanding the male-female differences in demographics, clinical and surgical characteristics and outcomes could modify the physician's practice and improve the quality of management.

\section{Author affiliations}

${ }^{1}$ Department of Anesthesiology, Chang Gung Memorial Hospital, Linkou Medical Center, Taoyuan, Taiwan

${ }^{2}$ Division of Thoracic and Cardiovascular Surgery, Department of Surgery, Chang Gung Memorial Hospital, Linkou Medical Center, Taoyuan, Taiwan

${ }^{3}$ Center for Big Data Analytics and Statistics, Chang Gung Memorial Hospital, Linkou Medical Center, Taoyuan, Taiwan

${ }^{4}$ Department of Cardiology, Chang Gung Memorial Hospital, Linkou Medical Center, Taoyuan, Taiwan

Acknowledgements We thank Alfred Hsing-Fen Lin and Ben Yu-Lin Chou for their assistance in the statistical analysis.

Contributors F-CC: conceptualisation; data curation; methodology; investigation; validation; writing —original draft. S-WC: conceptualisation; data curation; methodology; formal analysis; validation; writing—original draft. Y-HC: methodology; formal analysis; investigation; resources; writing — review and editing. C-PL: conceptualisation; formal analysis; data curation; resources; validation; writing — review and editing. VC-CW: data curation; formal analysis; writing — review and editing. Y-TC: conceptualisation; data curation; formal analysis; supervision. D-YC: conceptualisation; formal analysis; software. K$\mathrm{CH}$ : data curation; methodology; software. P-HC: methodology; data curation; resources; supervision; writing — review and editing. A-HC: conceptualisation; investigation; project administration; funding acquisition; validation; writingreview and editing. All authors give final approval of the version to be submitted and any revised version.

Funding The study was supported by a grant from the Chang Gung Medical Research Project (BMRPC19 , CMRPG3K1431, CFRPG3K0071). The funders had no role in the design of the study; in the collection, analyses, or interpretation of data; in the writing of the manuscript, or in the decision to publish the results.

Competing interests None declared.

Patient consent for publication Not applicable.

Ethics approval This cohort study was evaluated and approved via the NHIRD research committee and the institutional review board of Chang Gung Memorial Hospital and waived the need for individual informed consent (registration number: 104-7990B).

Provenance and peer review Not commissioned; externally peer reviewed.

Data availability statement No data are available.

Supplemental material This content has been supplied by the author(s). It has not been vetted by BMJ Publishing Group Limited (BMJ) and may not have been peer-reviewed. Any opinions or recommendations discussed are solely those of the author(s) and are not endorsed by BMJ. BMJ disclaims all liability and responsibility arising from any reliance placed on the content. Where the content includes any translated material, BMJ does not warrant the accuracy and reliability of the translations (including but not limited to local regulations, clinical guidelines, terminology, drug names and drug dosages), and is not responsible for any error and/or omissions arising from translation and adaptation or otherwise.

Open access This is an open access article distributed in accordance with the Creative Commons Attribution Non Commercial (CC BY-NC 4.0) license, which permits others to distribute, remix, adapt, build upon this work non-commercially, and license their derivative works on different terms, provided the original work is properly cited, appropriate credit is given, any changes made indicated, and the use is non-commercial. See: http://creativecommons.org/licenses/by-nc/4.0/.

\section{ORCID iDs}

Feng-Cheng Chang http://orcid.org/0000-0003-4947-2896

Pao-Hsien Chu http://orcid.org/0000-0003-0361-6348

An-Hsun Chou http://orcid.org/0000-0002-1711-5424

\section{REFERENCES}

1 Vaccarino V, Abramson JL, Veledar E, et al. Sex differences in hospital mortality after coronary artery bypass surgery: evidence for a higher mortality in younger women. Circulation 2002;105:1176-81.

2 Doenst T, Ivanov J, Borger MA, et al. Sex-specific long-term outcomes after combined valve and coronary artery surgery. Ann Thorac Surg 2006;81:1632-6.

3 Johnston A, Mesana TG, Lee DS, et al. Sex differences in long-term survival after major cardiac surgery: a population-based cohort study. J Am Heart Assoc 2019;8:e013260.

4 Buth KJ, Gainer RA, Legare J-F, et al. The changing face of cardiac surgery: practice patterns and outcomes 2001-2010. Can J Cardiol 2014;30:224-30.

5 Chaker Z, Badhwar V, Alqahtani F, et al. Sex differences in the utilization and outcomes of surgical aortic valve replacement for severe aortic stenosis. J Am Heart Assoc 2017;6 doi:10.1161/ JAHA.117.006370

6 Elhmidi Y, Piazza N, Mazzitelli D, et al. Sex-related differences in 2197 patients undergoing isolated surgical aortic valve replacement. J Card Surg 2014;29:772-8.

7 Mokhles MM, Siregar S, Versteegh MIM, et al. Male-female differences and survival in patients undergoing isolated mitral valve surgery: a nationwide cohort study in the Netherlands. Eur $J$ Cardiothorac Surg 2016;50:482-7.

8 Mokhles MM, Soloukey Tbalvandany S, Siregar S, et al. Male-female differences in aortic valve and combined aortic valve/coronary surgery: a national cohort study in the Netherlands. Open Heart 2018;5:e000868.

9 Onorati F, D'Errigo P, Barbanti M, et al. Different impact of sex on baseline characteristics and major periprocedural outcomes of transcatheter and surgical aortic valve interventions: results of the multicenter Italian OBSERVANT registry. J Thorac Cardiovasc Surg 2014;147:1529-39.

10 Veen KM, Mokhles MM, Braun J, et al. Male-female differences in characteristics and early outcomes of patients undergoing tricuspid valve surgery: a national cohort study in the Netherlands. Eur $J$ Cardiothorac Surg 2019;55:859-66.

11 Hsing AW, loannidis JPA. Nationwide population science: lessons from the Taiwan National health insurance research database. JAMA Intern Med 2015;175:1527-9. 
12 Chen S-W, Wu VC-C, Lin Y-S, et al. Propensity score matched analysis of mechanical vs. bioprosthetic valve replacement in patients with previous stroke. Circ J 2018;82:2041-8.

13 Lee H-A, Cheng Y-T, Wu VC-C, et al. Nationwide cohort study of mitral valve repair versus replacement for infective endocarditis. $J$ Thorac Cardiovasc Surg 2018;156:1473-83.

14 Wong W-K, Chen S-W, Chou A-H, et al. Late outcomes of valve repair versus replacement in isolated and concomitant tricuspid valve surgery: a nationwide cohort study. J Am Heart Assoc 2020;9:e015637.

15 Wu C-Y, Chen Y-J, Ho HJ, et al. Association between nucleoside analogues and risk of hepatitis $B$ virus-related hepatocellular carcinoma recurrence following liver resection. JAMA 2012;308:1906-14.

16 Faerber $\mathrm{G}$, Zacher M, Reents W, et al. Female sex is not a risk factor for post procedural mortality in coronary bypass surgery in the elderly: a secondary analysis of the GOPCABE trial. PLoS One 2017;12:e0184038.

17 Gupta S, Lui B, Ma X, et al. Sex differences in outcomes after coronary artery bypass grafting. J Cardiothorac Vasc Anesth 2020;34:3259-66.

18 Parvand M, Rayner-Hartley E, Sedlak T. Recent developments in sex-related differences in presentation, prognosis, and management of coronary artery disease. Can J Cardiol 2018;34:390-9.

19 Koch CG, Nussmeier NA. Gender and cardiac surgery. Anesthesiol Clin North Am 2003;21:675-89.

20 Aranki SF, Rizzo RJ, Couper GS, et al. Aortic valve replacement in the elderly. Effect of gender and coronary artery disease on operative mortality. Circulation 1993;88:li17-23.
21 Ibrahim MF, Paparella D, Ivanov J, et al. Gender-related differences in morbidity and mortality during combined valve and coronary surgery. J Thorac Cardiovasc Surg 2003;126:959-64.

22 Thourani VH, Weintraub WS, Craver JM, et al. Influence of concomitant CABG and urgent/emergent status on mitral valve replacement surgery. Ann Thorac Surg 2000;70:778-83.

23 Gehlot A, Mullany CJ, Ilstrup D, et al. Aortic valve replacement in patients aged eighty years and older: early and long-term results. $J$ Thorac Cardiovasc Surg 1996;111:1026-36.

24 O'Shaughnessy S, Tangel V, Dzotsi S. Non-white race/ethnicity and female sex are associated with increased allogeneic red blood cell transfusion in cardiac surgery patients: 2007-2018. J Cardiothorac Vasc Anesth 2021

25 Ter Woorst JF, Hoff AHT, van Straten AHM, et al. Impact of sex on the outcome of isolated aortic valve replacement and the role of different preoperative profiles. J Cardiothorac Vasc Anesth 2019;33:1237-43.

26 Vassileva CM, McNeely C, Mishkel G, et al. Gender differences in long-term survival of Medicare beneficiaries undergoing mitral valve operations. Ann Thorac Surg 2013;96:1367-73.

27 Mirabel M, lung B, Baron G, et al. What are the characteristics of patients with severe, symptomatic, mitral regurgitation who are denied surgery? Eur Heart J 2007;28:1358-65.

28 Chandrasekhar J, Dangas G, Mehran R. Valvular heart disease in women, differential remodeling, and response to new therapies. Curr Treat Options Cardiovasc Med 2017;19:74.

29 Cheng C-L, Chien H-C, Lee C-H, et al. Validity of in-hospital mortality data among patients with acute myocardial infarction or stroke in national health insurance research database in Taiwan. Int J Cardiol 2015;201:96-101. 